REPRESENTATION THEORY

An Electronic Journal of the American Mathematical Society

Volume 13, Pages 33-49 (February 23, 2009)

S 1088-4165(09)00345-8

\title{
A LIE-THEORETIC CONSTRUCTION OF SOME REPRESENTATIONS OF THE DEGENERATE AFFINE AND DOUBLE AFFINE HECKE ALGEBRAS OF TYPE $B C_{n}$
}

\author{
PAVEL ETINGOF, REBECCA FREUND, AND XIAOGUANG MA
}

\begin{abstract}
Let $G=G L(N), K=G L(p) \times G L(q)$, where $p+q=N$, and let $n$ be a positive integer. We construct a functor from the category of HarishChandra modules for the pair $(G, K)$ to the category of representations of the degenerate affine Hecke algebra of type $B_{n}$, and a functor from the category of $K$-monodromic twisted $D$-modules on $G / K$ to the category of representations of the degenerate double affine Hecke algebra of type $B C_{n}$; the second functor is an extension of the first one.
\end{abstract}

\section{INTRODUCTION}

The degenerate affine Hecke algebra (dAHA) of any Coxeter group was defined by Drinfeld and Lusztig (Dri, Lus ). It is generated by the group algebra of the Coxeter group and by the commuting generators $y_{i}$ with some relations.

The degenerate double affine Hecke algebra (dDAHA) of a root system was introduced by Cherednik (see $\mathrm{Ch}$ ). It is generated by the group algebra of the Weyl group, commuting generators $y_{i}$, and by another kind of commuting generators $X_{i}$ with some relations. The dAHA corresponding to the Weyl group can be realized as a subalgebra of dDAHA, generated by the Weyl group and the elements $y_{i}$.

The paper AS gives a Lie-theoretic construction of representations of the dAHA of type $A_{n-1}$. Namely (see CEE, Section 9 ), for every $\mathfrak{s l}_{N}$-bimodule $M$, an action of the dAHA $\mathcal{H}$ of type $A_{n-1}$ is constructed on the space

$$
F_{n}(M):=\left(M \otimes\left(\mathbb{C}^{N}\right)^{\otimes n}\right)^{\mathfrak{s l}_{N}},
$$

where the invariants are taken with respect to the adjoint action of $\mathfrak{s l}_{N}$ on $M$.

This construction is upgraded to a Lie-theoretic construction of representations of dDAHA of type $A_{n-1}$; see CEE, Section 9. Namely, for any $\mathcal{D}$-module $M$ on $S L_{N}$, the paper CEE] constructs an action of dDAHA $\mathcal{H}$ with parameter $k=N / n$ on the space $F_{n}(M)$, such that the induced action of the dAHA $\mathcal{H} \subset \mathcal{H}$ coincides with the action of $\mathrm{AS}$, obtained by regarding $M$ as an $\mathfrak{s l}_{N}$-bimodule via left- and right-invariant vector fields on $S L_{N}$.

The main result of this paper is an analog of the constructions of [AS] and CEE for dAHA and dDAHA of type $B C_{n}$, which gives a method of obtaining representations of these algebras from Lie theory. Specifically, given a module $M$ over the Lie algebra $\mathfrak{g}:=\mathfrak{g l}_{N}$, we first construct an action of the dAHA $\mathcal{H}$ of type $B_{n}$ on the space $F_{n, p, \mu}(M)$ of $\mu$-invariants in $M \otimes\left(\mathbb{C}^{N}\right)^{\otimes n}$ under the subalgebra

Received by the editors January 10, 2008 and, in revised form, October 14, 2008.

2000 Mathematics Subject Classification. Primary 16G99.

(C)2009 American Mathematical Society Reverts to public domain 28 years from publication 
$\mathfrak{k}_{0}:=\left(\mathfrak{g l}_{p} \oplus \mathfrak{g l}_{q}\right) \cap \mathfrak{s l}_{N} \subset \mathfrak{g}$, where $q=N-p$, and $\mu \in \mathbb{C}$ is a parameter (here by $\mu$-invariants we mean eigenvectors of $\mathfrak{k}_{0}$ with eigenvalues given by the character $\mu \chi$, where $\chi$ is a basic character of $\left.\mathfrak{k}_{0}\right)$. In this construction, the parameters of $\mathcal{H}$ are certain explicit functions of $\mu$ and $p$. Thus we obtain an exact functor $F_{n, p, \mu}$ from the category of $\mathfrak{g l}_{N}$-modules to the category of representations of $\mathcal{H}$. It is easy to see that this functor factors through the category of Harish-Chandra modules for the symmetric pair $\left(\mathfrak{g l}_{N}, \mathfrak{g l}_{p} \oplus \mathfrak{g l}_{q}\right)$, so it suffices to restrict our attention to Harish-Chandra modules.

Then we upgrade this construction to one giving representations of dDAHA $\mathcal{H}$ of type $B C_{n}$. Namely, let $G=G L_{N}$, and $K=G L_{p} \times G L_{q} \subset G$. Then for any $\lambda$-twisted $\mathcal{D}$-module $M$ on $G / K$ we construct an action of the dDAHA $\mathcal{H}$ of

type $B C_{n}$ on the space $F_{n, p, \mu}(M)$. In this construction, the parameters of $\mathcal{H}$ are certain explicit functions of $\lambda, \mu$, and $p$. Moreover, the underlying representation of $\mathcal{H}$ coincides with the representation obtained in the previous construction, if we regard $M$ as a $\mathfrak{g l}_{N}$-module via the vector fields corresponding to the action of $G$ on $G / K$. Thus we obtain an exact functor $F_{n, p, \mu}^{\lambda}$ from the category of $\lambda$-twisted $\mathcal{D}$-modules on $G / K$ to the category of representations of $\mathcal{H} \mathcal{H}$. This functor factors through the category of $K$-monodromic twisted $\mathcal{D}$-modules, so it suffices to restrict our attention to such $\mathcal{D}$-modules.

Since the appearance of the first version of this paper, the functor $F_{n, p, \mu}$ has been studied in the followup paper [M] by the third author. In this paper, it was shown that the principal series representations of $U(p, q)$ are mapped by the $F_{n, p, \mu}$ to certain induced modules over the dAHA. This is analogous to the result of [AS], where it is shown that in the type $A$ case, standard modules go to standard modules.

We expect that further careful study of the functors $F_{n, p, \mu}$ and $F_{n, p, \mu}^{\lambda}$ will reveal an interesting new connection between, on the one hand, the representation theory of (the universal cover of) the Lie group $U(p, q)$ and the theory of monodromic $\mathcal{D}$-modules on the (complexification of the) corresponding symmetric space $U(p, q) / U(p) \times U(q)$, and, on the other hand, the representation theory of dAHA and dDAHA of type $B C_{n}$. We plan to discuss this in future publications.

The paper is organized as follows. In Section 2, we recall the definitions of dAHA and dDAHA of a root system. In Section 3, we write down the explicit definitions for dAHA and dDAHA of type $B C_{n}$. In Section 4 , we construct the functor $F_{n, p, \mu}$. In Section 5, we construct the functor $F_{n, p, \mu}^{\lambda}$. In Section 6 , we study some properties of this functor.

\section{Definitions AND NOtATIONS}

In this section, we recall the definitions of degenerate affine and double affine Hecke algebras. For more details, see Ch.

Let $\mathfrak{h}$ be a finite-dimensional real vector space with a positive definite symmetric bilinear form $(\cdot, \cdot)$. Let $\left\{\epsilon_{i}\right\}$ be a basis for $\mathfrak{h}$ such that $\left(\epsilon_{i}, \epsilon_{j}\right)=\delta_{i j}$. Let $R$ be an irreducible root system in $\mathfrak{h}$ (possibly nonreduced). Let $R_{+}$be the set of positive roots of $R$, and let $\Pi=\left\{\alpha_{i}\right\}$ be the set of simple roots.

For any root $\alpha$, the corresponding coroot is $\alpha^{\vee}=2 \alpha /(\alpha, \alpha)$. Let $Q$ and $Q^{\vee}$ be the root lattice and the coroot lattice. Let $P=\operatorname{Hom}_{\mathbb{Z}}\left(Q^{\vee}, \mathbb{Z}\right)$ be the weight lattice.

Let $W$ be the Weyl group of $R$. Let $\Sigma$ be the set of reflections in $W$. Let $S_{\alpha} \in \Sigma$ be the reflection corresponding to the root $\alpha$. In particular, we write $S_{i}$ for the simple reflections $S_{\alpha_{i}}$. 
If $\alpha$ is a root, let $\nu_{\alpha}=1$, if $\alpha$ is an indivisible root, and $\nu_{\alpha}=2$ otherwise.

Let us define dAHA. Let $\kappa: \Sigma \rightarrow \mathbb{C}$ be a conjugation invariant function.

Definition 2.1. The degenerate affine Hecke algebra $(d A H A) \mathcal{H}(\kappa)$ is the quotient of the free product $\mathbb{C} W * S \mathfrak{h}$ by the relations

$$
S_{i} y-y^{S_{i}} S_{i}=\kappa\left(S_{i}\right) \alpha_{i}(y), \quad y \in \mathfrak{h} .
$$

For any $a \neq 0$, multiplication of $y$-generators by $a$ defines an isomorphism of $\mathcal{H}(\kappa)$ with $\mathcal{H}(a \kappa)$ (under this transformation, nothing happens to $\alpha_{i}(y)$ since they are just numbers). Thus in the simply laced situation, there is only one nontrivial case, $\kappa=1$, and in the nonsimply laced case, the function $\kappa$ takes two values $\kappa_{1}, \kappa_{2}$ (the values of $\kappa$ on the root reflections for long and short indivisible roots, respectively), and the algebra depends only on the ratio $\kappa_{2} / \kappa_{1}$ (unless both values are zero).

Now let us define dDAHA. Let $k: R \rightarrow \mathbb{C}, \alpha \mapsto k_{\alpha}$, be a function such that $k_{g(\alpha)}=k_{\alpha}$ for all $g \in W$. Let $t \in \mathbb{C}$.

Definition 2.2. For $\epsilon \in \mathfrak{h}$, define the Dunkl-Cherednik operator

$$
D_{\epsilon}(t, k)=t \partial_{\epsilon}-\sum_{\alpha \in R_{+}} \frac{k_{\alpha} \alpha(\epsilon)}{1-e^{-\alpha}}\left(1-S_{\alpha}\right)+\rho(k)(\epsilon),
$$

where $\partial_{\epsilon}$ is the differentiation along $\epsilon$, and $\rho(k)=\frac{1}{2} \sum_{\alpha \in R_{+}} k_{\alpha} \alpha$. This operator acts on the space $E$ of trigonometric polynomials on $\mathfrak{h} / Q^{\vee}$.

An important property of the operators $D_{\epsilon}$ is that they commute with each other.

Definition 2.3. The degenerate double affine Hecke algebra (dDAHA) $\mathcal{H}(t, k)$ is generated by $W$, the Dunkl-Cherednik operators, and the elements $e^{\lambda}(\lambda \in P)$.

Remark 2.4. This is not the original definition of dDAHA, but it is equivalent to the original definition by a theorem of Cherednik; see [Ch.

Obviously, for any $a \neq 0$, we have a natural isomorphism between $\mathcal{H}(t, k)$ and $\mathcal{H}(a t, a k)$; thus, there are only two essentially different cases: $t=0$ (the classical case) and $t=1$ (the quantum case).

The following proposition can be proved by a straightforward computation.

Proposition 2.5 (see $[\mathrm{Ch}]$ ). The subalgebra of the dDAHA $\mathcal{H}(t, k)$ generated by $W$ and the Dunkl-Cherednik operators is isomorphic to the dAHA $\mathcal{H}(\kappa)$, where $\kappa(S)=\sum_{\alpha: S=S_{\alpha}} k_{\alpha} \nu_{\alpha}$.

\section{TYPE $B C_{n} d$ AHA AND $d$ DAHA}

3.1. Definitions of the type $B C_{n}$ dAHA and dDAHA. Let us now describe the dAHA and dDAHA of type $B C_{n}$ more explicitly.

Let $\mathfrak{h}$ be a real vector space of dimension $n$ with orthonormal basis $\epsilon_{1}, \ldots, \epsilon_{n}$. We will identify $\mathfrak{h}$ with its dual by the bilinear form, and set $X_{i}=e^{\epsilon_{i}}, y_{i}=D_{\epsilon_{i}}$.

The roots of type $B C_{n}$ are

$$
R=\left\{ \pm \epsilon_{i}\right\} \cup\left\{ \pm 2 \epsilon_{i}\right\} \cup\left\{ \pm \epsilon_{i} \pm \epsilon_{j}\right\}_{i \neq j}
$$

and the positive roots are

$$
R_{+}=\left\{\epsilon_{i}\right\} \cup\left\{2 \epsilon_{i}\right\} \cup\left\{\epsilon_{i} \pm \epsilon_{j}\right\}_{i<j} .
$$


The function $\kappa$ considered in the previous section reduces to two parameters $\kappa=\left(\kappa_{1}, \kappa_{2}\right)$, while the function $k$ reduces to three parameters $k=\left(k_{1}, k_{2}, k_{3}\right)$ corresponding to the three kinds of positive roots: those of lengths 2,1 , 4 , respectively. Namely, $k_{1}=k_{\epsilon_{i}-\epsilon_{j}}, k_{2}=k_{\epsilon_{i}}, k_{3}=2 k_{2 \epsilon_{i}}$.

Let $W=S_{n} \ltimes(\mathbb{Z} / 2 \mathbb{Z})^{n}$ be the Weyl group of type $B C_{n}$. We denote by $S_{i j}$ the reflection in this group corresponding to the root $\epsilon_{i}-\epsilon_{j}$, and by $\gamma_{i}$ the reflection corresponding to $\epsilon_{i}$. Then $W$ is generated by $S_{i}=S_{i, i+1}, i=1, \ldots, n-1$ and $\gamma_{n}$.

The type $B C_{n}$ dAHA $\mathcal{H}\left(\kappa_{1}, \kappa_{2}\right)$ is then defined as follows:

- generators: $y_{1}, \ldots, y_{n}$ and $\mathbb{C} W$;

- relations:

i) $S_{i}$ and $\gamma_{n}$ satisfy the Coxeter relations;

ii) $S_{i} y_{i}-y_{i+1} S_{i}=\kappa_{1},\left[S_{i}, y_{j}\right]=0,(j \neq i, i+1)$;

iii) $\gamma_{n} y_{n}+y_{n} \gamma_{n}=\kappa_{2},\left[\gamma_{n}, y_{j}\right]=0,(j \neq n)$;

iv) $\left[y_{i}, y_{j}\right]=0$.

On the other hand, the type $B C_{n}$ dDAHA $\mathcal{H}\left(t, k_{1}, k_{2}, k_{3}\right)$ is defined as follows:

- generators: $X_{1}, \ldots, X_{n}, y_{1}, \ldots, y_{n}$ and $\mathbb{C} W$;

- relations:

i) $S_{i}$ and $\gamma_{n}$ satisfy the Coxeter relations;

ii) $S_{i} X_{i}-X_{i+1} S_{i}=0,\left[S_{i}, X_{j}\right]=0,(j \neq i, i+1)$;

iii) $S_{i} y_{i}-y_{i+1} S_{i}=k_{1},\left[S_{i}, y_{j}\right]=0,(j \neq i, i+1)$;

iv) $\gamma_{n} y_{n}+y_{n} \gamma_{n}=k_{2}+k_{3}, \gamma_{n} X_{n}=X_{n}^{-1} \gamma_{n}$, $\left[\gamma_{n}, y_{j}\right]=\left[\gamma_{n}, X_{j}\right]=0,(j \neq n)$

v) $\left[X_{i}, X_{j}\right]=\left[y_{i}, y_{j}\right]=0$;

vi) $\left[y_{j}, X_{i}\right]=k_{1} X_{i} S_{i j}-k_{1} X_{i} S_{i j} \gamma_{i} \gamma_{j},\left[y_{i}, X_{j}\right]$ vii) $=k_{1} X_{i} S_{i j}-k_{1} X_{j} S_{i j} \gamma_{i} \gamma_{j},(i<j)$;

$$
\begin{aligned}
{\left[y_{i}, X_{i}\right]=} & t X_{i}-k_{1} X_{i} \sum_{k>i} S_{i k}-k_{1} \sum_{k<i} S_{i k} X_{i}-k_{1} X_{i} \sum_{k \neq i} S_{i k} \gamma_{i} \gamma_{k} \\
& -\left(k_{2}+k_{3}\right) X_{i} \gamma_{i}-k_{2} \gamma_{i} .
\end{aligned}
$$

In particular, we see that the subalgebra in the dDAHA generated by $W$ and $y_{i}$ is $\mathcal{H}\left(\kappa_{1}, \kappa_{2}\right)$, where $\kappa_{1}=k_{1}$ and $\kappa_{2}=k_{2}+k_{3}$.

3.2. Another set of generators of dAHA of type $B C_{n}$. The advantage of the generators $y_{i}$ is that they commute with each other, but their disadvantage is that they do not change according to the standard representation of the Weyl group. It turns out that it is possible (and useful in some situations, including one of this paper) to trade the first property for the second one, by replacing the generators $y_{i}$ by their shifted versions $\tilde{y}_{i}$, passing from Lusztig's presentation of dAHA ([Lus] to one of Drinfeld's ([Dri $]$ ) 1

Namely, for each $i=1, \ldots, n$, define

$$
\tilde{y}_{i}=y_{i}-\frac{\kappa_{2}}{2} \gamma_{i}-\frac{\kappa_{1}}{2} \sum_{k>i} S_{i k}+\frac{\kappa_{1}}{2} \sum_{k<i} S_{i k}-\frac{\kappa_{1}}{2} \sum_{i \neq k} S_{i k} \gamma_{i} \gamma_{k} .
$$

Lemma 3.1. The type $B C_{n} d A H A \mathcal{H}\left(\kappa_{1}, \kappa_{2}\right)$ is generated by $w \in W$ and $\tilde{y}_{i}$ with the following relations:

i) $S_{i} \tilde{y}_{i}-\tilde{y}_{i+1} S_{i}=0, \quad S_{j} \tilde{y}_{i}-\tilde{y}_{i} S_{j}=0,(i \neq j)$;

\footnotetext{
${ }^{1}$ See $[\mathrm{RS}]$ for more details.
} 
ii) $\tilde{y}_{n} \gamma_{n}+\gamma_{n} \tilde{y}_{n}=0, \quad \tilde{y}_{i} \gamma_{n}-\gamma_{n} \tilde{y}_{i}=0,(i \neq n)$;

iii)

$$
\begin{aligned}
{\left[\tilde{y}_{i}, \tilde{y}_{j}\right]=} & \frac{\kappa_{1} \kappa_{2}}{2} S_{i j}\left(\gamma_{j}-\gamma_{i}\right)+\frac{\kappa_{1}^{2}}{4} \sum_{k \neq i, j} S_{j k} S_{i k}-\frac{\kappa_{1}^{2}}{4} \sum_{k \neq i, j} S_{i k} S_{j k} \\
+ & \frac{\kappa_{1}^{2}}{4} \sum_{k \neq i, j} S_{i k} S_{j k}\left(-\gamma_{i} \gamma_{k}+\gamma_{i} \gamma_{j}+\gamma_{j} \gamma_{k}\right) \\
& -\frac{\kappa_{1}^{2}}{4} \sum_{k \neq i, j} S_{j k} S_{i k}\left(\gamma_{i} \gamma_{j}-\gamma_{j} \gamma_{k}+\gamma_{i} \gamma_{k}\right) .
\end{aligned}
$$

Proof. The proof is contained in the proof of Lemma 3.2 given in the next subsection.

3.3. Another set of generators of dDAHA of type $B C_{n}$. Similarly to the previous subsection, for each $i=1, \ldots, n$, define

$$
\tilde{y}_{i}=y_{i}-\frac{k_{2}+k_{3}}{2} \gamma_{i}-\frac{k_{1}}{2} \sum_{k>i} S_{i k}+\frac{k_{1}}{2} \sum_{k<i} S_{i k}-\frac{k_{1}}{2} \sum_{i \neq k} S_{i k} \gamma_{i} \gamma_{k}
$$

(together with the Weyl group, the elements $\tilde{y}_{i}$ generate dAHA in Drinfeld's presentation [Dri]).

Lemma 3.2. The type $B C_{n} d D A H A \mathfrak{H}\left(t, k_{1}, k_{2}, k_{3}\right)$ is generated by $w \in W, X_{i}$ and $\tilde{y}_{i}$ with the following relations:

i) the relations among elements of $W$ and $X_{i}$ are the same as before;

ii) $S_{i} \tilde{y}_{i}-\tilde{y}_{i+1} S_{i}=0, \quad S_{j} \tilde{y}_{i}-\tilde{y}_{i} S_{j}=0,(i \neq j)$;

iii) $\tilde{y}_{n} \gamma_{n}+\gamma_{n} \tilde{y}_{n}=0, \quad \tilde{y}_{i} \gamma_{n}-\gamma_{n} \tilde{y}_{i}=0,(i \neq n)$;

iv) $\left[\tilde{y}_{j}, X_{i}\right]=\frac{k_{1}}{2}\left(X_{i}+X_{j}\right) S_{i j}-\frac{k_{1}}{2}\left(X_{i}+X_{j}^{-1}\right) S_{i j} \gamma_{i} \gamma_{j},(i \neq j)$;

v)

$$
\begin{aligned}
{\left[\tilde{y}_{i}, X_{i}\right]=} & t X_{i}-\frac{k_{2}+k_{3}}{2}\left(X_{i}^{-1}+X_{i}\right) \gamma_{i}-k_{2} \gamma_{i} \\
& -\frac{k_{1}}{2} \sum_{k \neq i}\left(\left(X_{i}+X_{k}\right) S_{i k}+\left(X_{i}+X_{k}^{-1}\right) S_{i k} \gamma_{i} \gamma_{k}\right)
\end{aligned}
$$

vi)

$$
\begin{aligned}
{\left[\tilde{y}_{i}, \tilde{y}_{j}\right]=} & \frac{k_{1}\left(k_{2}+k_{3}\right)}{2} S_{i j}\left(\gamma_{j}-\gamma_{i}\right)+\frac{k_{1}^{2}}{4} \sum_{k \neq i, j} S_{j k} S_{i k}-\frac{k_{1}^{2}}{4} \sum_{k \neq i, j} S_{i k} S_{j k} \\
+ & \frac{k_{1}^{2}}{4} \sum_{k \neq i, j} S_{i k} S_{j k}\left(-\gamma_{i} \gamma_{k}+\gamma_{i} \gamma_{j}+\gamma_{j} \gamma_{k}\right) \\
& -\frac{k_{1}^{2}}{4} \sum_{k \neq i, j} S_{j k} S_{i k}\left(\gamma_{i} \gamma_{j}-\gamma_{j} \gamma_{k}+\gamma_{i} \gamma_{k}\right),(i \neq j) .
\end{aligned}
$$

Proof. Only the last relation is nontrivial. Its proof is by a direct computation. To make formulas more compact, set

$$
R_{i}=-\frac{k_{1}}{2} \sum_{k>i} S_{i k}+\frac{k_{1}}{2} \sum_{k<i} S_{i k}-\frac{k_{1}}{2} \sum_{i \neq k} S_{i k} \gamma_{i} \gamma_{k}
$$


then we have

$$
y_{i}=\tilde{y}_{i}+\frac{k_{2}+k_{3}}{2} \gamma_{i}-R_{i} .
$$

Assume $i<j$, then we have

$$
\begin{aligned}
{\left[y_{i}, y_{j}\right]=} & {\left[\tilde{y}_{i}, \tilde{y}_{j}\right]-\left[\tilde{y}_{i}, R_{j}\right]-\left[R_{i}, \tilde{y}_{j}\right] } \\
& -\frac{k_{2}+k_{3}}{2}\left(\left[\gamma_{i}, R_{j}\right]+\left[R_{i}, \gamma_{j}\right]\right)+\left[R_{i}, R_{j}\right] .
\end{aligned}
$$

Since

$$
\begin{aligned}
& {\left[\tilde{y}_{i}, R_{j}\right]=\frac{k_{1}}{2} S_{i j} \tilde{y}_{j}-\frac{k_{1}}{2} S_{i j} \tilde{y}_{i}-\frac{k_{1}}{2} S_{i j} \tilde{y}_{j} \gamma_{i} \gamma_{j}-\frac{k_{1}}{2} S_{i j} \tilde{y}_{i} \gamma_{i} \gamma_{j},} \\
& {\left[R_{i}, \tilde{y}_{j}\right]=\frac{k_{1}}{2} S_{i j} \tilde{y}_{i}-\frac{k_{1}}{2} S_{i j} \tilde{y}_{j}+\frac{k_{1}}{2} S_{i j} \tilde{y}_{j} \gamma_{i} \gamma_{j}+\frac{k_{1}}{2} S_{i j} \tilde{y}_{i} \gamma_{i} \gamma_{j},} \\
& {\left[\gamma_{i}, R_{j}\right]=k_{1} S_{i j} \gamma_{j}-k_{1} S_{i j} \gamma_{i},} \\
& {\left[R_{i}, \gamma_{j}\right]=0}
\end{aligned}
$$

we have

$$
\left[\tilde{y}_{i}, \tilde{y}_{j}\right]=\frac{k_{1}\left(k_{2}+k_{3}\right)}{2} S_{i j}\left(\gamma_{j}-\gamma_{i}\right)-\left[R_{i}, R_{j}\right] .
$$

By direct computation, we have $\left[R_{i}, R_{j}\right]=\frac{k_{1}^{2}}{4} R_{i j}$, where

$$
\begin{aligned}
& R_{i j}=\sum_{k>j} S_{i j} S_{j k}-\sum_{k>j} S_{j k} S_{i j}+\sum_{k>j} S_{i k} S_{j k}-\sum_{k>j} S_{j k} S_{i k} \\
& -\sum_{\substack{i<k<j \\
\text { or } k>j}} S_{i k} S_{i j}+\sum_{\substack{i<k<j \\
\text { or } k>j}} S_{i j} S_{i k}-\sum_{\substack{k<i \text { or } \\
i<k<j}} S_{i j} S_{j k}+\sum_{\substack{k<i \text { or } \\
i<k<j}} S_{j k} S_{i j} \\
& -\sum_{i<k<j} S_{i k} S_{j k}+\sum_{i<k<j} S_{j k} S_{i k}+\sum_{\begin{array}{c}
i<k<j \\
\text { or } k>j
\end{array}} S_{i k} S_{i j} \gamma_{i} \gamma_{j} \\
& -\sum_{\substack{i<k<j \\
\text { or } k>j}} S_{i j} S_{i k} \gamma_{j} \gamma_{k}+\sum_{\substack{k<i \text { or } \\
i<k<j \\
\text { or } k>j}} S_{i j} S_{j k} \gamma_{j} \gamma_{k}-\sum_{\substack{k<i \text { or } \\
i<k<j \\
\text { or } k>j}} S_{j k} S_{i j} \gamma_{i} \gamma_{k} \\
& +\sum_{\substack{i<k<j \\
\text { or } k>j}} S_{i k} S_{j k} \gamma_{j} \gamma_{k}-\sum_{\substack{i<k<j \\
\text { or } k>j}} S_{j k} S_{i k} \gamma_{i} \gamma_{j} \\
& +\sum_{k<i} S_{i k} S_{i j}-\sum_{k<i} S_{i j} S_{i k}+\sum_{k<i} S_{i k} S_{j k}-\sum_{k<i} S_{j k} S_{i k} \\
& -\sum_{k<i} S_{i k} S_{i j} \gamma_{i} \gamma_{j}+\sum_{k<i} S_{i j} S_{i k} \gamma_{j} \gamma_{k}-\sum_{k<i} S_{i k} S_{j k} \gamma_{j} \gamma_{k}+\sum_{k<i} S_{j k} S_{i k} \gamma_{i} \gamma_{j} \\
& +\sum_{k>j} S_{i j} S_{j k} \gamma_{i} \gamma_{k}-\sum_{k>j} S_{j k} S_{i j} \gamma_{i} \gamma_{j}+\sum_{k>j} S_{i k} S_{j k} \gamma_{i} \gamma_{j}-\sum_{k>j} S_{j k} S_{i k} \gamma_{i} \gamma_{k} \\
& -\sum_{\substack{k<i \text { or } \\
i<k<j \\
\text { or } k>j}} S_{i k} S_{i j} \gamma_{j} \gamma_{k}+\sum_{\substack{k<i \text { or } \\
i<k<j \\
\text { or } k>j}} S_{i j} S_{i k} \gamma_{i} \gamma_{k}-\sum_{\substack{k<i \text { or } \\
i<k<j}} S_{i j} S_{j k} \gamma_{i} \gamma_{k}
\end{aligned}
$$




$$
\begin{aligned}
& +\sum_{\substack{k<i \text { or } \\
i<k<j}} S_{j k} S_{i j} \gamma_{i} \gamma_{j}-\sum_{\substack{k<i \text { or } \\
i<k<j}} S_{i k} S_{j k} \gamma_{i} \gamma_{j}+\sum_{\substack{k<i \text { or } \\
i<k<j}} S_{j k} S_{i k} \gamma_{i} \gamma_{k} \\
+ & \sum_{k \neq i, j} S_{i k} S_{i j} \gamma_{i} \gamma_{k}-\sum_{k \neq i, j} S_{i j} S_{i k} \gamma_{i} \gamma_{j}+\sum_{k \neq i, j} S_{i j} S_{j k} \gamma_{i} \gamma_{j} \\
& -\sum_{k \neq i, j} S_{j k} S_{i j} \gamma_{j} \gamma_{k}+\sum_{k \neq i, j} S_{i k} S_{j k} \gamma_{i} \gamma_{k}-\sum_{k \neq i, j} S_{j k} S_{i k} \gamma_{j} \gamma_{k} \\
= & -\sum_{k \neq i, j} S_{j k} S_{i k}+\sum_{k \neq i, j} S_{i k} S_{j k} \\
& -\sum_{k \neq i, j} S_{i k} S_{j k}\left(-\gamma_{i} \gamma_{k}-\gamma_{i} \gamma_{j}+\gamma_{j} \gamma_{k}\right)+\sum_{k \neq i, j} S_{j k} S_{i k}\left(\gamma_{i} \gamma_{j}-\gamma_{j} \gamma_{k}+\gamma_{i} \gamma_{k}\right) .
\end{aligned}
$$

Thus, from the commutativity of $y_{i}$ and the above, we get

$$
\begin{gathered}
{\left[\tilde{y}_{i}, \tilde{y}_{j}\right]=\frac{k_{1}\left(k_{2}+k_{3}\right)}{2} S_{i j}\left(\gamma_{j}-\gamma_{i}\right)+\frac{k_{1}^{2}}{4} \sum_{k \neq i, j} S_{j k} S_{i k}-\frac{k_{1}^{2}}{4} \sum_{k \neq i, j} S_{i k} S_{j k}} \\
+\frac{k_{1}^{2}}{4} \sum_{k \neq i, j} S_{i k} S_{j k}\left(-\gamma_{i} \gamma_{k}+\gamma_{i} \gamma_{j}+\gamma_{j} \gamma_{k}\right) \\
\quad-\frac{k_{1}^{2}}{4} \sum_{k \neq i, j} S_{j k} S_{i k}\left(\gamma_{i} \gamma_{j}-\gamma_{j} \gamma_{k}+\gamma_{i} \gamma_{k}\right),
\end{gathered}
$$

as desired.

\section{Construction of the functor $F_{n, p, \mu}$}

4.1. Notations. We will use the following notations. Let $p, q \in \mathbb{N}$ and let $N=p+q$. Let $E_{i j}$ be the $N$ by $N$ matrix which has a 1 at the $(i, j)$-th position and 0 elsewhere. Set $J=\left(\begin{array}{ll}I_{p} & \\ & -I_{q}\end{array}\right)$, where $I_{p}$ is the identity matrix of size $p$.

Let $\mathfrak{g}=\mathfrak{g l}_{N}(\mathbb{C})$ be the Lie algebra of $G=G L_{N}(\mathbb{C})$. Let $\mathfrak{k}=\mathfrak{g l}_{p}(\mathbb{C}) \times \mathfrak{g l}_{q}(\mathbb{C})$ be the Lie algebra of $K=G L_{p}(\mathbb{C}) \times G L_{q}(\mathbb{C})$. Let $\mathfrak{k}_{0}$ be the subalgebra of trace zero matrices in $\mathfrak{k}$.

Define a character $\chi$ of $\mathfrak{k}$ by

$$
\chi\left(\left(\begin{array}{cc}
X_{1} & 0 \\
0 & X_{2}
\end{array}\right)\right)=q \operatorname{tr} X_{1}-p \operatorname{tr} X_{2} .
$$

For this $\chi$, we have the following obvious lemma.

Lemma 4.1. We have $\chi\left(E_{i j}\right)=0$ for $i \neq j$, and

$$
\chi\left(E_{i i}\right)=\left\{\begin{array}{cc}
q, & i \leq p \\
-p, & i>p .
\end{array}\right.
$$

In particular, $\chi\left(I_{N}\right)=0$.

Remark 4.2. The property $\chi\left(I_{N}\right)=0$ is the reason why we choose such $\chi$. 
We will also use the following summation notations:

$$
\begin{aligned}
& \sum_{i, \ldots, j}=\sum_{i=1}^{n} \ldots \sum_{j=1}^{n}, \quad \sum_{i \ldots j}=\sum_{i=1}^{p} \ldots \sum_{j=1}^{p}+\sum_{i=p+1}^{n} \ldots \sum_{j=p+1}^{n}, \\
& \sum_{i \ldots j \mid k \ldots l}=\sum_{i=1}^{p} \cdots \sum_{j=1}^{p} \sum_{k=p+1}^{n} \cdots \sum_{l=p+1}^{n}+\sum_{k=1}^{p} \cdots \sum_{l=1}^{p} \sum_{i=p+1}^{n} \ldots \sum_{j=p+1}^{n} .
\end{aligned}
$$

Thus we have two ranges of summation $([1, p]$ and $[p+1, N])$, and the indices not separated by anything must be in the same range, while indices separated by a vertical line must be in different ranges. Indices separated by a comma are independent.

4.2. Construction of the functor $F_{n, p, \mu}$. Let $Y$ be a $\mathfrak{k}_{0}$-module. For any $\mu \in \mathbb{C}$, define the space of $\mu$-invariants $Y^{\mathfrak{k}_{0}, \mu}$ to be the space of those $v \in Y$ for which $x v=\mu \chi(x) v$ for all $x \in \mathfrak{k}_{0}$.

Let $V=\mathbb{C}^{N}$ be the vector representation of $\mathfrak{g}$. Let $M$ be a $\mathfrak{g}$-module. Define

$$
F_{n, p, \mu}(M)=\left(M \otimes V^{\otimes n}\right)^{\mathfrak{k}_{0}, \mu} .
$$

The Weyl group $W$ acts on $M \otimes V^{\otimes n}$ in the following way: the element $S_{i j}$ acts by exchanging the $i$-th and $j$-th factors, and $\gamma_{i}$ acts by multiplying the $i$-th factor by $J$ (here we regard $M$ as the 0 -th factor). Thus we have a natural action of $W$ on $F_{n, p, \mu}(M)$.

Define elements $\tilde{y}_{k} \in \operatorname{End}\left(F_{n, p, \mu}(M)\right)$ as follows:

$$
\tilde{y}_{k}=-\sum_{i \mid j} E_{i j} \otimes\left(E_{j i}\right)_{k}, \text { for } k=1, \ldots, n,
$$

where the first component acts on $M$ and the second component acts on the $k$-th factor of the tensor product.

The main result of this section is the following theorem.

Theorem 4.3. The above action of $W$ and the elements $\tilde{y}_{k}$ given by (1) combine into a representation of the degenerate affine Hecke algebra $\mathcal{H}\left(\kappa_{1}, \kappa_{2}\right)$ (in the presentation of Lemma 3.1) on the space $F_{n, p, \mu}(M)$, with

$$
\kappa_{1}=1, \kappa_{2}=p-q-\mu N .
$$

So we have an exact functor $F_{n, p, \mu}$ from the the category of $\mathfrak{g}$-modules to the category of representations of type $B C_{n} d A H A$ with such parameters.

Proof. Our job is to show that the elements $\tilde{y}_{k}, S_{k}$ and $\gamma_{n}$ satisfy the relations in Lemma 3.1. We only need to prove the commutation relation between the elements $\tilde{y}_{k}$, since the other relations are trivial.

Let $a \neq b$ and $\delta_{i j}$ be the identity matrix, then we have

$$
\begin{aligned}
& {\left[\tilde{y}_{a}, \tilde{y}_{b}\right]} \\
& \quad=\sum_{i l \mid j} E_{i l} \otimes\left(E_{j i}\right)_{a} \otimes\left(E_{l j}\right)_{b}-\sum_{i \mid k j} E_{k j} \otimes\left(E_{j i}\right)_{a} \otimes\left(E_{i k}\right)_{b} \\
& \quad=\sum_{i l \mid j}\left(E_{i l}-\frac{I_{N}}{N} \delta_{i l}\right) \otimes\left(E_{j i}\right)_{a} \otimes\left(E_{l j}\right)_{b}-\sum_{i \mid k j}\left(E_{k j}-\frac{I_{N}}{N} \delta_{k j}\right) \otimes\left(E_{j i}\right)_{a} \otimes\left(E_{i k}\right)_{b}
\end{aligned}
$$

(by the $\mu$-invariance and Lemma 4.1) 


$$
\begin{aligned}
= & ((q-p)+\mu N)\left(\sum_{i \leq p, j>p} 1 \otimes\left(E_{j i}\right)_{a} \otimes\left(E_{i j}\right)_{b}-\sum_{i>p, j \leq p} 1 \otimes\left(E_{j i}\right)_{a} \otimes\left(E_{i j}\right)_{b}\right) \\
& -\sum_{a \neq c \neq b}\left(\sum_{i l \mid j} 1 \otimes\left(E_{j i}\right)_{a} \otimes\left(E_{l j}\right)_{b} \otimes\left(E_{i l}\right)_{c}-\sum_{i \mid k j} 1 \otimes\left(E_{j i}\right)_{a} \otimes\left(E_{i k}\right)_{b} \otimes\left(E_{k j}\right)_{c}\right) \\
= & \frac{p-q-\mu N}{2} S_{a b}\left(\gamma_{b}-\gamma_{a}\right)-\frac{1}{4} \sum_{a \neq c \neq b}\left(1-\gamma_{a} \gamma_{b}-\gamma_{a} \gamma_{c}+\gamma_{b} \gamma_{c}\right) S_{a c} S_{b c} \\
& +\frac{1}{4} \sum_{a \neq c \neq b}\left(1-\gamma_{a} \gamma_{b}+\gamma_{a} \gamma_{c}-\gamma_{b} \gamma_{c}\right) S_{b c} S_{a c} .
\end{aligned}
$$

Comparing this to the relation in Lemma 3.1 we get the result.

4.3. Example. Consider the example $p=q=1, N=2$. Thus, $\kappa_{1}=1, \kappa_{2}=-\mu N$. For the module $M$, let us take the module $\mathcal{F}_{\lambda, \nu}$ of tensor fields $p(z) z^{\nu / 2}(d z / z)^{\lambda}$, where $p$ is a Laurent polynomial; the Lie algebra $\mathfrak{g l}_{2}$ acts on it by infinitesimal fractional linear transformations of $z$. Then we get $F_{n, p, \mu}\left(\mathcal{F}_{\lambda, \mu-n}\right)=Y_{\lambda}$, a representation of $\mathcal{H}\left(\kappa_{1}, \kappa_{2}\right)$ of dimension $2^{n}$, which is isomorphic to $V^{\otimes n}$ as a $W$-module. The structure of $Y_{\lambda}$ as a dAHA-module is discussed in the recent paper [M].

\section{Construction of the functor $F_{n, p, \mu}^{\lambda}$}

5.1. The main theorem. Let $\lambda \in \mathbb{C}$. For $x \in \mathfrak{g}$, let $L_{x}$ denote the vector field on $G$ generated by the left action of $x$. Thus, $\left(L_{x} f\right)(A)=\left.\frac{d}{d t}\right|_{t=0} f\left(e^{t x} A\right)$ for a function $f$. Note that $L_{[x, y]}=-\left[L_{x}, L_{y}\right]$ (the minus sign comes from the fact that left multiplication by elements of $G$ gives rise to a right action of $G$ on functions on $G)$.

Let $\mathcal{D}^{\lambda}(G / K)$ be the sheaf of differential operators on $G / K$, twisted by the character $\lambda \chi$. Local sections of $\mathcal{D}^{\lambda}(G / K)$ act naturally on $\lambda \chi$-twisted functions on $G / K$, i.e., analytic functions $f$ on a small open set $U \subset G$ such that $R_{z} f=$ $\lambda \chi(z) f, z \in \mathfrak{k}$, where $R_{z}$ is the left invariant vector field corresponding to the right translation by $z$. This action is faithful. Note that we can regard elements $L_{x}$ as global sections of $\mathcal{D}^{\lambda}(G / K)$, with the same commutation law $\left[L_{x}, L_{y}\right]=-L_{[x, y]}$.

Let $M$ be a $\mathcal{D}^{\lambda}(G / K)$-module. Then $M$ is naturally a $\mathfrak{g}$-module, via the vector fields $L_{x}$. Define

$$
F_{n, p, \mu}^{\lambda}(M)=\left(M \otimes V^{\otimes n}\right)^{\mathfrak{k}_{0}, \mu} .
$$

Then $F_{n, p, \mu}^{\lambda}(M)$ is a $W$-module as in the previous section.

For $k=1, \ldots, n$, define the following linear operators on the space $F_{n, p, \mu}^{\lambda}(M)$ :

$$
X_{k}=\sum_{i, j}\left(A J A^{-1} J\right)_{i j} \otimes\left(E_{i j}\right)_{k}, \quad \tilde{y}_{k}=\sum_{i \mid j} L_{i j} \otimes\left(E_{j i}\right)_{k},
$$

where $\left(A J A^{-1} J\right)_{i j}$ is the function of $A \in G / K$ which takes the $i j$-th element of $A J A^{-1} J, L_{i j}=L_{E_{i j}}$, and the second component acts on the $k$-th factor in $V^{\otimes n}$. From now on, we write $X=A J A^{-1} J$ and $X^{-1}=J A J A^{-1}$. Thus, we have $J X=X^{-1} J$.

The main result of this section is the following theorem.

Theorem 5.1. The above action of $W$ and the elements in (3) combine into a representation of the degenerate double affine Hecke algebra $\mathfrak{H}\left(t, k_{1}, k_{2}, k_{3}\right)$ (in the 
presentation of Lemma 3.2) on the space $F_{n, p, \mu}^{\lambda}(M)$, with

$$
t=\frac{2 n}{N}+(\lambda+\mu)(q-p), \quad k_{1}=1, \quad k_{2}=p-q-\lambda N, \quad k_{3}=(\lambda-\mu) N .
$$

So we have an exact functor $F_{n, p, \mu}^{\lambda}$ from the the category of $\mathcal{D}^{\lambda}(G / K)$-modules to the category of representations of the type $B C_{n} d D A H A$ with such parameters.

Note that the restriction of the representation $F_{n, p, \mu}^{\lambda}(M)$ to the affine Hecke algebra $\mathcal{H}$ clearly coincides with the representation of Theorem 4.3 .

Remark. This construction is parallel to the similar construction in type $A$ performed in CEE. The most important difference is that here we use the functions $\left(A J A^{-1} J\right)_{i j}$ on $G / K$ instead of the functions $A_{i j}$ on $G$ used in CEE. The motivation for this is that the matrix elements of $A J A^{-1} J$ are "the simplest" nonconstant algebraic functions on $G / K$, similarly to how the matrix elements of $A$ are "the simplest" nonconstant algebraic functions on $G$.

The rest of this section is devoted to the proof of Theorem 5.1.

5.2. Proof of Theorem 5.1. Our job is to show that the elements $X_{k}, \tilde{y}_{k}, S_{k}$ and $\gamma_{n}$ satisfy the relations in Lemma 3.2

First of all, the relations in Lemma 3.2 which do not involve $X_{i}$ can be established as in the proof of Theorem 4.3 (as (2) is compatible with (4)).

Second, there are some trivial relations:

$$
\begin{gathered}
{\left[X_{i}, X_{j}\right]=0, \quad\left[\gamma_{i}, X_{j}\right]=0, \quad(i \neq j),} \\
{\left[S_{i}, X_{j}\right]=0, \quad(j \neq i, i+1), \quad S_{i} X_{i}-X_{i+1} S_{i}=0,} \\
{\left[\gamma_{i}, \tilde{y}_{j}\right]=0, \quad(j \neq i), \quad \gamma_{i} \tilde{y}_{i}+\tilde{y}_{i} \gamma_{i}=0,}
\end{gathered}
$$

and since $J X=X^{-1} J$, we have

$$
\gamma_{i} X_{i}=X_{i}^{-1} \gamma_{i}
$$

Third, we have the following result.

Lemma 5.2. We have the following commutation relations: if $m \neq k$, then

$$
\begin{aligned}
{\left[\tilde{y}_{m}, X_{k}\right] } & =\frac{1}{2}\left(X_{k}+X_{m}\right) S_{m k}-\frac{1}{2}\left(X_{k}+X_{m}^{-1}\right) S_{m k} \gamma_{m} \gamma_{k}, \\
{\left[\tilde{y}_{m}, X_{k}^{-1}\right] } & =-\frac{1}{2}\left(X_{k}^{-1}+X_{m}^{-1}\right) S_{m k}+\frac{1}{2}\left(X_{k}^{-1}+X_{m}\right) S_{m k} \gamma_{m} \gamma_{k} .
\end{aligned}
$$

So we have

$\left[\tilde{y}_{m}, X_{k}+X_{k}^{-1}\right]=\frac{1}{2}\left(X_{k}-X_{k}^{-1}+X_{m}-X_{m}^{-1}\right) S_{m k}+\frac{1}{2}\left(X_{k}^{-1}-X_{k}+X_{m}-X_{m}^{-1}\right) S_{m k} \gamma_{m} \gamma_{k}$.

Proof. The proof is by direct computation. First, we have for $r \leq p<s$ or $s \leq p<r$

$$
L_{r s}(X)_{i j}=\delta_{s j}(X)_{i r}+\delta_{r i}(X)_{s j}
$$


So

$$
\begin{aligned}
& {\left[\tilde{y}_{m}, X_{k}\right]} \\
& \quad=\sum_{r \mid s} \sum_{i, j} L_{r s}(X)_{i j} \otimes\left(E_{s r}\right)_{m} \otimes\left(E_{i j}\right)_{k} \\
& =\sum_{r \mid s} \sum_{i}(X)_{i r} \otimes\left(E_{s r}\right)_{m} \otimes\left(E_{i s}\right)_{k}+\sum_{r \mid s} \sum_{j}(X)_{s j} \otimes\left(E_{s r}\right)_{m} \otimes\left(E_{r j}\right)_{k} \\
& =\frac{1}{2}\left(X_{k}+X_{m}\right) S_{m k}-\frac{1}{2}\left(X_{k}+X_{m}^{-1}\right) S_{m k} \gamma_{m} \gamma_{k} .
\end{aligned}
$$

Using a similar method, we can obtain the other identities.

Thus, we only need to show that $X_{m}$ and $\tilde{y}_{m}$ satisfy v) in Lemma 3.2 if the parameters satisfy (4). Instead of computing $\left[\tilde{y}_{m}, X_{m}\right]$, we will compute $\left[\tilde{y}_{m}, X_{m}+\right.$ $\left.X_{m}^{-1}\right]$ and $\left[\tilde{y}_{m}, X_{m}-X_{m}^{-1}\right]$.

5.2.1. Computing $\left[\tilde{y}_{m}, X_{m}+X_{m}^{-1}\right]$. Let us define

$$
T=\operatorname{tr}(X)=\sum_{i}(X)_{i i} \otimes 1 .
$$

Suppose $X=\left(\begin{array}{ll}A_{1} & A_{2} \\ A_{3} & A_{4}\end{array}\right)$ where $A_{1}$ is a $p$ by $p$ matrix. Then

$$
\operatorname{tr}\left(A_{1}\right)=\operatorname{tr}(X(J+1) / 2), \quad \operatorname{tr}\left(A_{4}\right)=\operatorname{tr}(X(1-J) / 2) .
$$

But $\operatorname{tr}(X J)=\operatorname{tr}\left(A J A^{-1}\right)=\operatorname{tr}(J)=p-q$, so we get

$$
\operatorname{tr}\left(A_{1}\right)=\frac{T+p-q}{2}, \quad \operatorname{tr}\left(A_{4}\right)=\frac{T-p+q}{2} .
$$

Lemma 5.3. We have the relation

$$
\sum_{m}\left(X_{m}+X_{m}^{-1}\right)=\left(\frac{2 n}{N}+(\lambda+\mu)(q-p)\right) T+(\lambda+\mu)\left(p^{2}-q^{2}\right) .
$$

Proof. Since $(X)_{i j}=-\left(X^{-1}\right)_{i j}$ unless $i, j \leq p$ or $i, j>p$, and $(X)_{i j}=\left(X^{-1}\right)_{i j}$ if $i, j \leq p$ or $i, j>p$, we have

$$
X_{m}+X_{m}^{-1}=\sum_{i j}\left(X+X^{-1}\right)_{i j} \otimes\left(E_{i j}\right)_{m} .
$$

Thus, we have

$$
\begin{aligned}
& \sum_{m}\left(X_{m}+X_{m}^{-1}\right) \\
& \quad=\sum_{m} \sum_{i j}\left(X+X^{-1}\right)_{i j} \otimes\left(E_{i j}-\frac{I_{N}}{N} \delta_{i j}\right)_{m}+\sum_{m} \sum_{i}\left(X+X^{-1}\right)_{i i} \otimes\left(\frac{I_{N}}{N}\right)_{m}
\end{aligned}
$$

(by the $\mu$-invariance and Lemma 4.1)

$$
=Y+\frac{2 n}{N} T+\sum_{i \leq p} \mu q\left(X+X^{-1}\right)_{i i} \otimes 1-\sum_{i>p} \mu p\left(X+X^{-1}\right)_{i i} \otimes 1
$$

(by (6) )

$$
=Y+\left(\frac{2 n}{N}+\mu(q-p)\right) T+\mu\left(p^{2}-q^{2}\right),
$$

where $Y=\sum_{i j}\left(X+X^{-1}\right)_{i j} L_{E_{i j}} \otimes 1$. 
It remains to calculate the expression $Y$ in the algebra $\mathcal{D}^{\lambda}(G / K)$. We can calculate $Y$ by acting with it on $\lambda \chi$-twisted functions $f$ on $G / K$.

We have

$$
\begin{aligned}
(Y f)(A) & =\left.\frac{d}{d t}\right|_{t=0} f\left(A+t\left(X+X^{-1}\right) A\right) \\
& =\left.\frac{d}{d t}\right|_{t=0} f\left(A+t A J A^{-1} J A+t J A J\right) \\
& =\left.\frac{d}{d t}\right|_{t=0} f\left(A+t A\left(J A^{-1} J A+A^{-1} J A J\right)\right) \\
& =\left.\frac{d}{d t}\right|_{t=0} f\left(A+t A\left(X_{*}+X_{*}^{-1}\right)\right),
\end{aligned}
$$

where $X_{*}:=J A^{-1} J A$. Now, $X_{*}+X_{*}^{-1} \in \mathfrak{k}$, so we have

$$
Y f=\lambda \chi\left(X_{*}+X_{*}^{-1}\right) f=\lambda\left((q-p) T+\left(p^{2}-q^{2}\right)\right) f .
$$

Combining this with formula (7), we obtain the statement of the lemma.

Notice that

$$
\begin{aligned}
{\left[\tilde{y}_{m}, T\right] } & =\sum_{r \mid s} L_{r s}(\operatorname{tr}(X)) \otimes\left(E_{s r}\right)_{m} \\
& =\sum_{r \mid s}\left((X)_{s r}-\left(X^{-1}\right)_{s r}\right) \otimes\left(E_{s r}\right)_{m} \\
& =X_{m}-X_{m}^{-1} .
\end{aligned}
$$

So from Lemma 5.2 and Lemma 5.3. we have

$$
\left[\tilde{y}_{m}, X_{m}+X_{m}^{-1}\right]=-\sum_{k \neq m}\left[\tilde{y}_{m}, X_{k}+X_{k}^{-1}\right]+\left(\frac{2 n}{N}+(\lambda+\mu)(q-p)\right)\left[\tilde{y}_{m}, T\right] .
$$

Thus, we have obtained

\section{Lemma 5.4.}

$$
\begin{aligned}
{\left[\tilde{y}_{m}, X_{m}+X_{m}^{-1}\right]=} & \left(\frac{2 n}{N}+(\lambda+\mu)(q-p)\right)\left(X_{m}-X_{m}^{-1}\right) \\
& -\frac{1}{2} \sum_{k \neq m}\left(X_{k}-X_{k}^{-1}+X_{m}-X_{m}^{-1}\right) S_{m k} \\
& +\frac{1}{2} \sum_{k \neq m}\left(X_{k}-X_{k}^{-1}-X_{m}+X_{m}^{-1}\right) S_{m k} \gamma_{m} \gamma_{k} .
\end{aligned}
$$

5.2.2. Computing $\left[\tilde{y}_{m}, X_{m}-X_{m}^{-1}\right]$. At first, we need the following lemmas for the future computation.

Lemma 5.5. We have the equality

$$
\begin{gathered}
q \sum_{s \leq p} \sum_{j}\left(X+X^{-1}\right)_{s j} \otimes\left(E_{s j}\right)_{m}+p \sum_{s>p} \sum_{j}\left(X+X^{-1}\right)_{s j} \otimes\left(E_{s j}\right)_{m} \\
=\frac{1}{2}\left(N+(q-p) \gamma_{m}\right)\left(X_{m}+X_{m}^{-1}\right) .
\end{gathered}
$$

Proof. By direct computation. 
Lemma 5.6. In $\mathcal{D}^{\lambda}(G / K)$, we have for $r, j \leq p$ or $r, j>p$,

$$
L_{[X-X}^{\left.-1, E_{r j}\right]}=-L_{\left\{X+X^{-1}, E_{r j}\right\}}+2 \lambda \chi\left(Q_{r j}\right),
$$

where $\{a, b\}=a b+b a$ and $Q_{r j}=J A^{-1} J E_{r j} A+A^{-1} E_{r j} J A J$.

Proof. Let $f(A)$ be a $\lambda \chi$-twisted function on $G / K$, i.e., an analytic function on a small open set $U \subset G$ such that $R_{z} f=\lambda \chi(z) f, z \in \mathfrak{k}$. Then we have

$$
\begin{aligned}
& L_{\left[X-X^{-1}, E_{r j}\right]} f(A) \\
& \quad=\left.\frac{d}{d t}\right|_{t=0} f\left(A+t\left(X E_{r j}+E_{r j} X^{-1}\right) A-t\left(X^{-1} E_{r j}+E_{r j} X\right) A\right) .
\end{aligned}
$$

Note that

$$
\begin{aligned}
f(A+ & \left.t\left(X E_{r j}+E_{r j} X^{-1}\right) A-t\left(X^{-1} E_{r j}+E_{r j} X\right) A\right) \\
& =f\left(A+2 t A Q_{r j}-t\left(X E_{r j}+E_{r j} X^{-1}\right) A-t\left(X^{-1} E_{r j}+E_{r j} X\right) A\right),
\end{aligned}
$$

and $Q_{r j}$ is an element of $\mathfrak{k}$.

So we have

$$
\begin{aligned}
\left.\frac{d}{d t}\right|_{t=0} f\left(A+t A Q_{r j}-t\left(X^{-1} E_{r j}+E_{r j} X\right) A\right) \\
=\left.\frac{d}{d t}\right|_{t=0} f\left(A-t\left(X^{-1} E_{r j}+E_{r j} X\right) A-t\left(X E_{r j}+E_{r j} X^{-1}\right) A\right) \\
\quad+\left.\frac{d}{d t}\right|_{t=0} f\left(A+2 t A Q_{r j}\right) \\
=-L_{\left\{X+X^{-1}, E_{r j}\right\}} f(A)+2 \lambda \chi\left(Q_{r j}\right) f(A) .
\end{aligned}
$$

Thus we get the lemma.

Now let us compute $\left[\tilde{y}_{m}, X_{m}-X_{m}^{-1}\right]$. By the definition and Lemma [5.6, we have

$$
\begin{aligned}
& {\left[\tilde{y}_{m}, X_{m}-X_{m}^{-1}\right]} \\
& =\sum_{r \mid s} \sum_{i, j} L_{r s}\left((X)_{i j}-\left(X^{-1}\right)_{i j}\right) \otimes\left(E_{s r} E_{i j}\right)_{m} \\
& \quad-\sum_{r j} L_{\left\{X+X^{-1}, E_{j r}\right\}} \otimes\left(E_{r j}\right)_{m} \\
& \quad+2 \lambda \sum_{r j} \chi\left(J A^{-1} J E_{r j} A+A^{-1} E_{r j} J A J\right) \otimes\left(E_{j r}\right)_{m} .
\end{aligned}
$$

Since we have

$$
L_{r s}\left((X)_{i j}-\left(X^{-1}\right)_{i j}\right)=(X)_{i r} \delta_{s j}+(X)_{s j} \delta_{i r}+\left(X^{-1}\right)_{i r} \delta_{s j}+\left(X^{-1}\right)_{s j} \delta_{i r}
$$

by Lemma 5.5 , the first summand of (9) is

$\frac{1}{2}\left(N+(q-p) \gamma_{m}\right)\left(X_{m}+X_{m}^{-1}\right)+\left(1+\gamma_{m}\right) \frac{T-p+q}{2}+\left(1-\gamma_{m}\right) \frac{T+p-q}{2}$.

Now let us compute the second summand of (9). By definition, we have

$$
\begin{aligned}
& -\sum_{r j} L_{\left\{X+X^{-1}, E_{r j}\right\}} \otimes\left(E_{j r}\right)_{m} \\
& =-\sum_{i j r}\left(\left(X+X^{-1}\right)_{i r} L_{E_{i j}}+\left(X+X^{-1}\right)_{j i} L_{E_{r i}}\right) \otimes\left(E_{j r}\right)_{m}
\end{aligned}
$$




$$
\begin{aligned}
= & -\sum_{i j r}\left(\left(X+X^{-1}\right)_{i r} L_{E_{i j}-\frac{I_{N}}{N} \delta_{i j}}+\left(X+X^{-1}\right)_{j i} L_{E_{r i}-\frac{I_{N}}{N} \delta_{r i}}\right) \otimes\left(E_{j r}\right)_{m} \\
= & -\sum_{i j r}\left(X+X^{-1}\right)_{i r} \otimes\left(E_{j r} E_{i j}\right)_{m}-\sum_{k \neq m} \sum_{i j r}\left(X+X^{-1}\right)_{i r} \otimes\left(E_{i j}\right)_{k} \otimes\left(E_{j r}\right)_{m} \\
- & \sum_{i j r}\left(X+X^{-1}\right)_{j i} \otimes\left(E_{j r} E_{r i}\right)_{m}-\sum_{k \neq m} \sum_{i j r}\left(X+X^{-1}\right)_{j i} \otimes\left(E_{r i}\right)_{k} \otimes\left(E_{j r}\right)_{m} \\
+ & \sum_{i j r}\left(X+X^{-1}\right)_{i r} \otimes\left(E_{j r} \frac{I_{N}}{N} \delta_{i j}\right)_{m}+\sum_{k \neq m} \sum_{i j r}\left(X+X^{-1}\right)_{i r} \otimes\left(\frac{I_{N}}{N} \delta_{i j}\right)_{k} \otimes\left(E_{j r}\right)_{m} \\
+ & \sum_{i j r}\left(X+X^{-1}\right)_{j i} \otimes\left(E_{j r} \frac{I_{N}}{N} \delta_{r i}\right)_{m}+\sum_{k \neq m} \sum_{i j r}\left(X+X^{-1}\right)_{j i} \otimes\left(\frac{I_{N}}{N} \delta_{r i}\right)_{k} \otimes\left(E_{j r}\right)_{m} \\
+ & \mu\left((q-p)+N \gamma_{m}\right)\left(X_{m}+X_{m}^{-1}\right) \\
= & -T+(q-p) \gamma_{m}-\frac{1}{2}\left(X_{m}+X_{m}^{-1}\right)\left(N+(p-q) \gamma_{m}\right) \\
& -\frac{1}{2} \sum_{k \neq m}\left(X_{m}+X_{m}^{-1}+X_{k}+X_{k}^{-1}\right) S_{k m}\left(1+\gamma_{k} \gamma_{m}\right) \\
& +\frac{2 n\left(X_{m}+X_{m}^{-1}\right)}{N}+\mu\left((q-p)+N \gamma_{m}\right)\left(X_{m}+X_{m}^{-1}\right) .
\end{aligned}
$$

Now let us compute the third summand of (9).

Lemma 5.7. For $r, j \leq p$ or $r, j>p$, we have

$$
\begin{aligned}
& \lambda \chi\left(J A^{-1} J E_{r j} A+A^{-1} E_{r j} J A J\right) \\
& \quad= \begin{cases}\frac{\lambda(q-p)}{2}\left(X+X^{-1}\right)_{j r}+\lambda N \delta_{r j}, & r, j \leq p, \\
\frac{\lambda(q-p)}{2}\left(X+X^{-1}\right)_{j r}-\lambda N \delta_{r j}, & r, j>p .\end{cases}
\end{aligned}
$$

Proof. Let us denote

$$
B=A^{-1} E_{r j} J A=\left(\begin{array}{ll}
B_{1} & B_{2} \\
B_{3} & B_{4}
\end{array}\right) \text {, where } B_{1} \text { is a } p \text { by } p \text { matrix. }
$$

Then

$$
J A^{-1} J E_{r j} A+A^{-1} E_{r j} J A J=J B+B J=\left(\begin{array}{cc}
2 B_{1} & \\
& -2 B_{4}
\end{array}\right) .
$$

Then we have

$$
2 \operatorname{tr}\left(B_{1}\right)-2 \operatorname{tr}\left(B_{4}\right)=\left(X+X^{-1}\right)_{j r} .
$$

On the other hand, we have

$$
\operatorname{tr}(B)=\operatorname{tr}\left(B_{1}\right)+\operatorname{tr}\left(B_{4}\right)=\operatorname{tr}\left(A^{-1} E_{r j} J A\right)=\left\{\begin{array}{cc}
\delta_{r j}, & r j \leq p, \\
-\delta_{r j}, & r j>p .
\end{array}\right.
$$

Then we have for $j, r \leq p$,

for $j, r>p$,

$$
\operatorname{tr}\left(B_{1}\right)=\frac{1}{4}\left(X+X^{-1}\right)_{j r}+\frac{1}{2} \delta_{r j}, \quad \operatorname{tr}\left(B_{4}\right)=-\frac{1}{4}\left(X+X^{-1}\right)_{j r}+\frac{1}{2} \delta_{r j},
$$

$$
\operatorname{tr}\left(B_{1}\right)=\frac{1}{4}\left(X+X^{-1}\right)_{j r}-\frac{1}{2} \delta_{r j}, \quad \operatorname{tr}\left(B_{4}\right)=-\frac{1}{4}\left(X+X^{-1}\right)_{j r}-\frac{1}{2} \delta_{r j} .
$$

So we get the lemma. 
From Lemma 5.7, we have

$$
\begin{gathered}
\sum_{r j} \chi\left(J A^{-1} J E_{r j} A+A^{-1} E_{r j} J A J\right) \otimes\left(E_{j r}\right)_{m} \\
=\frac{1}{2}(q-p)\left(X_{m}+X_{m}^{-1}\right)+N \gamma_{m} .
\end{gathered}
$$

Thus, combining the above formulas, we have

\section{Lemma 5.8.}

$$
\begin{aligned}
{\left[\tilde{y}_{m}, X_{m}-X_{m}^{-1}\right]=} & \left(\frac{2 n}{N}+(\lambda+\mu)(q-p)\right)\left(X_{m}+X_{m}^{-1}\right) \\
& -\frac{1}{2} \sum_{k \neq m}\left(X_{k}+X_{k}^{-1}\right)\left(1+\gamma_{m} \gamma_{k}\right) S_{k m} \\
& -\frac{1}{2} \sum_{k \neq m}\left(X_{m}+X_{m}^{-1}\right)\left(1+\gamma_{m} \gamma_{k}\right) S_{k m} \\
& +((q-p)+\mu N) \gamma_{m}\left(X_{m}+X_{m}^{-1}\right)+2((q-p)+\lambda N) \gamma_{m}
\end{aligned}
$$

5.2.3. Conclusion. Adding equations (10) and (8), and comparing with Lemma 3.2. we conclude the proof of Theorem 5.1

\section{ACtion of $F_{n, p, \mu}^{\lambda}$ ON SOME SUbCATEGories}

As we mentioned, the functors $F_{n, p, \mu}, F_{n, p, \mu}^{\lambda}$ factor through the category of modules $M$ on which the action of $\mathfrak{k}$ is locally finite; more precisely, $F_{n, p, \mu}(M)=$ $F_{n, p, \mu}\left(M_{f}\right)$, where $M_{f}$ is the locally finite part of $M$ under $\mathfrak{k}$.

Now let $M$ be a $\mathcal{D}^{\lambda}(G / K)$-module, locally finite under $\mathfrak{k}$. The support of such a $\mathcal{D}$-module is a $K$-invariant subset of $G / K$, i.e., a union of $K$-orbits. Recall that closed $K$-orbits of $G / K$ are labeled by the points of the categorical quotient $K \backslash G / K$, i.e., the spectrum of the ring $R_{p, q}=\mathcal{O}(G / K)^{K}$. For every point $\psi \in$ $K \backslash G / K\left(\psi: R_{p, q} \rightarrow \mathbb{C}\right)$, we can define the subcategory $D^{\lambda}(\psi)$ of the category of $\mathcal{D}^{\lambda}$-modules on $G / K$ which are set-theoretically supported on the preimage of $\psi$ in $G / K$, i.e., those on which $R_{p, q}$ acts with generalized eigenvalue $\psi$.

On the other hand, let $\mathbb{T}=\mathbb{C}^{n} / \mathbb{Z}^{n}=\mathfrak{h} / Q^{\vee}$, and let $\beta \in \mathbb{T} / W$. Then we can define the category $\mathcal{O}_{\beta}$ of modules over the dDAHA $\mathscr{H}$ on which the subalgebra $\mathbb{C}[P]^{W} \subset \mathcal{H}$ acts with generalized eigenvalue $\beta$.

The following theorem tells us how the functor $F_{n, p, \mu}^{\lambda}$ relates $\psi$ and $\beta$.

Theorem 6.1. The functor $F_{n, p, \mu}^{\lambda}$ maps $D^{\lambda}(\psi)$ to $\mathcal{O}_{\beta}$, where $\beta=\theta(\psi)$, and $\theta$ : $K \backslash G / K \rightarrow \mathbb{T} / W$ is the regular map defined by the formula

$$
\theta^{*}\left(\sum_{m=1}^{n} g\left(X_{m}\right)\right)=n g(1)+\left(\frac{n}{N}+\frac{1}{2}(\lambda+\mu)(q-p)\right) \operatorname{tr}(g(X)-g(1)),
$$

where $g$ is a Laurent polynomial in one variable such that $g(Z)=g\left(Z^{-1}\right)$.

Proof. The proof is obtained by generalizing the proof of Lemma 5.3. We'll need the following lemma.

Lemma 6.2. $\operatorname{tr}\left(X^{s} J\right)=p-q$ for any $s \in \mathbb{Z}$.

Proof. It is easy to see that $X^{s} J$ is conjugate to $J$. 
The lemma implies that

$$
\operatorname{tr}\left(X^{s}(J+1) / 2\right)=\frac{\operatorname{tr}\left(X^{s}\right)+p-q}{2}
$$

and

$$
\operatorname{tr}\left(X^{s}(1-J) / 2\right)=\frac{\operatorname{tr}\left(X^{s}\right)-p+q}{2}
$$

Thus we have

$$
\begin{aligned}
& \sum_{m} g\left(X_{m}\right) \\
& \quad=\sum_{m} \sum_{i j} g(X)_{i j} \otimes\left(E_{i j}-\frac{I_{N}}{N} \delta_{i j}\right)_{m}+\sum_{m} \sum_{i} g(X)_{i i} \otimes\left(\frac{I_{N}}{N}\right)_{m}
\end{aligned}
$$

(by the $\mu$-invariance and Lemma 4.1)

$$
\begin{aligned}
& =Y_{g}+\frac{n}{N} \operatorname{tr}(g(X))+\sum_{i \leq p} \mu q g(X)_{i i} \otimes 1-\sum_{i>p} \mu p g(X)_{i i} \otimes 1 \\
& =Y_{g}+\left(\frac{n}{N}+\frac{1}{2} \mu(q-p)\right) \operatorname{tr}(g(X))+\frac{1}{2} \mu\left(p^{2}-q^{2}\right) g(1),
\end{aligned}
$$

where $Y_{g}=\sum_{i j} g(X)_{i j} L_{E_{i j}} \otimes 1$.

It remains to calculate the expression $Y_{g}$ in the algebra $\mathcal{D}^{\lambda}(G / K)$. We can calculate $Y_{g}$ by acting with it on $\lambda \chi$-twisted functions $f$ on $G / K$.

We have

$$
\begin{aligned}
\left(Y_{g} f\right)(A) & =\left.\frac{d}{d t}\right|_{t=0} f(A+\operatorname{tg}(X) A) \\
& =\left.\frac{d}{d t}\right|_{t=0} f\left(A+\operatorname{tAg}\left(X_{*}\right)\right)
\end{aligned}
$$

where $X_{*}:=J A^{-1} J A$. Now, $X_{*}+X_{*}^{-1} \in \mathfrak{k}$, so we have

$$
Y_{g} f=\lambda \chi\left(g\left(X_{*}\right)\right) f=\frac{1}{2} \lambda\left((q-p) \operatorname{tr}(g(X))+\frac{1}{2}\left(p^{2}-q^{2}\right) g(1)\right) f
$$

Combining this with formula (11), we obtain the statement of the theorem.

Remark 6.3. In particular, Theorem 6.1 implies that $\theta(1)=1$, where $1 \in K \backslash G / K$ is the double coset of 1 , and $1 \in \mathbb{T} / W$ is the image of the unit of the group $\mathbb{T}$. Thus the functor $F_{n, p, \mu}^{\lambda}$ maps the category $D^{\lambda}(1)$ to the category $\mathcal{O}_{1}$. Note that $D^{\lambda}(1)$ is the category of twisted $\mathcal{D}$-modules supported on the "unipotent variety" in $G / K$ (which is equivalent to the category on $\mathcal{D}$-modules on $\mathfrak{g} / \mathfrak{k}$ supported on the nilpotent cone), and $\mathcal{O}_{1}$ is the category of $\mathcal{H}\left(\right.$-modules on which $X_{i}$ act unipotently (which is equivalent to category $\mathcal{O}$ for the rational Cherednik algebra of type $B_{n}$ ).

Remark 6.4. Another very interesting question is how the functor $F_{n, p, \mu}$ transforms the central characters, i.e., how the central character of a Harish-Chandra module $M$ is related to the central character of the dAHA module $F_{n, p, \mu}(M)$. This question is discussed in the paper [M] (for $n=1$ ). 


\section{ACKNOWLEDGMENTS}

The work of the first author was partially supported by the NSF grant DMS0504847. The work of the second and the third authors was supported by the Summer Program of Undergraduate Research in the Department of Mathematics at MIT. We thank Ju-Lee Kim, David Vogan, and Ting Xue for useful discussions.

\section{REFERENCES}

[AS] T. Arakawa, T. Suzuki, Duality between $\mathfrak{s l}_{n}(\mathbb{C})$ and the degenerate affine Hecke algebra, J. Algebra 209 (1998), no. 1, 288-304. MR1652134(99h:17005)

[CEE] D. Calaque, B. Enriquez, P. Etingof, Universal KZB equations I: the elliptic case, arXiv:math/0702670.

[Ch] I. Cherednik, Double Affine Hecke Algebras, London Math. Soc. Lect. Note Ser. 319, Cambridge University, 2005. MR2133033 (2007e:32012)

[Dri] V. Drinfeld, Degenerate affine Hecke algebras and Yangians (Russian), Funktsional. Anal. i Prilozhen. 20 (1986), no. 1, 69-70. MR831053 (87m:22044)

[Lus] G. Lusztig, Affine Hecke algebra and their graded version, J. Amer. Math. Soc. 2 (1989), no. 3, 599-635. MR991016 (90e:16049)

[M] X. Ma, On some representations of degenerate affine Hecke algebras of type $B C_{n}$, arXiv:0810.0791.

[RS] A. Ram, A.V. Shepler, Classification of graded Hecke algebras for complex reflection groups, Comment. Math. Helv. 78 (2003), no. 2, 308-334. MR.1988199 (2004d:20007)

Department of Mathematics, Massachusetts Institute of Technology, Cambridge, Massachusetts 02139

E-mail address: etingof@math.mit.edu

Massachusetts Institute of Technology, Cambridge, Massachusetts 02139

E-mail address: rlfreund@mit.edu

Department of Mathematics, Massachusetts Institute of Technology, Cambridge, MASSACHUSETTS 02139

E-mail address: xma@math.mit.edu 